\title{
MUJERES EN LA ALTA DIRECCIÓN DE EDUCACIÓN SUPERIOR: POSIBILIDADES, TENSIONES Y NUEVAS INTERROGANTES
}

\section{La presencia femenina en los más altos niveles de decisión: un marco contextual introductorio}

En las últimas décadas hemos progresado de manera significativa en la igualdad entre los géneros. En términos globales, el acceso de las mujeres a la educación superior y la participación femenina en la fuerza de trabajo han aumentado. Sin embargo, no hemos sido capaces de eliminar la segregación ocupacional, las brechas salariales entre hombres y mujeres, ni el reparto desigual del trabajo doméstico y familiar (Parella, 2003). La realidad muestra que las mujeres concurren a los empleos menos cualificados, peor pagados y menos valorados, con una escasa presencia en puestos directivos y de mayor responsabilidad (Maruani, 2000). En América Latina, la participación laboral masculina en cargos de dirección duplica e incluso triplica a la femenina: las mujeres latinoamericanas representan el 31,1\% de los directivos de gobierno y empresas; el 48,8\% de los profesionales, científicos e intelectuales; el 59,3\% de los empleos de oficina; el $65 \%$ de los trabajadores de servicio y vendedores, y el $71 \%$ de los trabajadores no calificados (Papadópulos y Radakovich, 2006).

Si bien las mujeres chilenas han ingresado masivamente a la educación superior, alcanzado la mitad de las matrículas anuales de pregrado (Correa \& Monckeberg, 2001), no han tenido aún la oportunidad de capitalizar los logros educacionales accediendo a posiciones estratégicas y de liderazgo en la fuerza de trabajo de las esferas culturales, económicas, académicas y políticas de la sociedad chilena. Es aquí donde surge la llamada "paradoja de la mujer chilena" (Monserrat y Saracostti, 2006). Un segundo ángulo de esta paradoja se refleja en la incoherencia entre las creencias acerca de las 
oportunidades femeninas para alcanzar posiciones directivas y sus logros. Al respecto, se cree que la mujer chilena podría ser: "presidenta de la corte suprema (93,8\% de los entrevistados), presidenta de una gran compañía (99\%), presidenta de la República $(86,5 \%)$ y comandante en jefe del Ejército (74,2\%) (Fundación Futuro, 2003). Los alcances reales no se condicen con estas "creencias", lo cual se refleja en una participación insuficiente en los espacios de decisión.

De acuerdo con datos del Servicio Nacional de la Mujer (SERNAM) (2005), "Por cada 9 parlamentarios, hay 1 parlamentaria. Por cada 7 alcaldes, hay 1 alcaldesa. Por cada 4 concejales, hay 1 concejala. Por cada 20 ministros de la Corte Suprema, hay 1 ministra. Por cada 3 hombres en la directiva de la Central Unitaria de Trabajadores (CUT), hay 1 mujer. Por cada 5 hombres en las directivas de los partidos políticos, hay 1 mujer".

Estos datos reflejan una subrepresentación de mujeres en posiciones directivas: una exclusión de las esferas tradicionales del poder. El término "cielo de vidrio" describiría esa barrera invisible que permite a las mujeres ver la cima de diversas organizaciones, pero que las frena para alcanzarlas. En la actualidad, las mejores organizaciones se sustentan sobre una mezcla equilibrada de los denominados atributos "femeninos" y "masculinos". Asimismo, son cada día más las organizaciones que adoptan medidas para atraer y retener a las mujeres en diversas posiciones, incluidos los puestos directivos, para beneficiarse así de sus calificaciones y de su talento en un entorno cada vez más competitivo (Wirth, 2002). Este documento es una invitación a reflexionar respecto de las posibilidades y dificultades que enfrentan las mujeres chilenas para alcanzar posiciones estratégicas en las distintas facetas de la vida pública, en general, y en las instituciones de educación superior, en particular.

\section{Aclaraciones metodológicas}

Este artículo se sustenta en un estudio de carácter exploratorio respecto de las experiencias de mujeres que han alcanzado posiciones 
directivas (decanas, vicerrectoras o rectoras) en cuatro instituciones chilenas de educación superior. El objetivo general de este estudio ha sido indagar acerca de las posibilidades y tensiones experimentadas por mujeres que han alcanzado posiciones de poder en instituciones de educación superior. Para tal efecto, se ha utilizado una metodología de investigación cualitativa, ya que refiere a "una investigación que produce datos descriptivos: las propias palabras de las personas, habladas o escritas, y la conducta observable". (Taylor y Bogdan, 1992). La necesidad de una corriente epistemológica nos acerca a la perspectiva fenomenológica que se basa en la convicción de que "la conducta humana, lo que la gente dice y hace, es producto del modo en que define su mundo" (Taylor y Bogdan, 1992: 16), entendiendo entonces los fenómenos sociales y culturales desde la experiencia de quienes los viven, de cómo los significan y revelan.

Como instrumento para conocer las percepciones de los sujetos, y dentro de los mecanismos de recolección de información para la investigación cualitativa, se utilizarán las entrevistas en profundidad, las cuales "siguen el modelo de una conversación entre iguales y no de un intercambio formal de preguntas y respuestas" (Taylor y Bogdan, 1992).

Se han definido una serie de interrogantes relativas a las posibilidades y limitaciones de las mujeres de acceder y desarrollarse en posiciones directivas en las instituciones de educación superior. ¿Por qué existen tan pocas mujeres en cargos directivos en este ámbito? ¿Cómo entienden y gestionan el poder las mujeres que se han desempeñado como decanas, vicerrectoras o rectoras? ¿Existen diferencias en sus concepciones y gestión del poder en relación con los varones? ¿Cuáles son las dificultades a que se enfrentan? Reflexionar acerca de estas interrogantes ha implicado indagar en un conjunto de dimensiones constituyentes en la generación del poder al interior de estas instituciones, para lo cual se entrevistó a un grupo selecto de mujeres que se han desempeñado como decanas, vicerrectoras académicas y rectoras tanto de universidades del Consejo de Rectores de Universidades Chilenas (CRUCH) como de entidades privadas. 


\section{Mujeres en la alta dirección de educación superior: la situación en números}

No es de extrañar que en una institución como la universitaria, surgida en la Edad Media con el renacer del mundo griego, persista un discurso invisible contra las mujeres que imperaron en la Grecia clásica (Guil, 2002). Efectivamente, podía leerse en 1377, en los estatutos de la Universidad de Bolonia: "Y puesto que la mujer es la razón primera del pecado, el arma del demonio, la causa de la expulsión del hombre del paraíso y de la destrucción de la antigua ley, y puesto que, en consecuencia, es preciso evitar cuidadosamente todo comercio con ella, nosotros defendemos y prohibimos expresamente que alguien se permita introducir alguna mujer, sea cual fuere ésta, incluso la más honrada, en la dicha universidad. Y si alguno lo hace a pesar de todo, será severamente castigado por el rector" (tomado de Lerena, 1976: 299).

Desde la Edad Media a la fecha la situación laboral de la mujer y el contexto sociopolítico han cambiado bastante. La historia reciente muestra la presencia de mujeres en distintos ámbitos de la vida social, incluidas las instituciones de educación superior. Hombres y mujeres tienen niveles de matrícula similares en estas entidades. Sin embargo, las mujeres han alcanzado una presencia limitada en posiciones directivas (jefas de carrera, decanas, vicerrectoras o rectoras). "... No es una cosa tan extraña el que haya pocas mujeres en cargos de poder universitario, está enmarcado dentro de lo que pasa en el país..." (entrevistada 1) Como se muestra en la tabla 1, durante 2005, el 16\% de los rectores de instituciones de educación superior (universidades, institutos profesionales y centros de formación profesional) eran mujeres. Más específicamente en el caso de las universidades, en 2000 sólo cuatro mujeres -equivalente al 6,25\% del total de rectores del país- se desempeñaba como rectoras de universidades, mientras que en 2005 este número ha ascendido marginalmente a cinco rectoras -equivalente al $8 \%$ del total de rectores del país. 
Tabla 1: Rectores según género (INDICES 2005).

\begin{tabular}{l|r|r|r|r|r|r}
\hline & \multicolumn{2}{c}{ Hombres } & \multicolumn{2}{c}{ Mujeres } & \multicolumn{2}{c}{ Total } \\
\hline Universidades & 57 & $92 \%$ & 5 & $8 \%$ & 62 & $45 \%$ \\
\hline Institutos profesionales & 27 & $79 \%$ & 7 & $21 \%$ & 34 & $25 \%$ \\
\hline Centros de formación técnica & 31 & $76 \%$ & 10 & $24 \%$ & 41 & $30 \%$ \\
\hline Total & 115 & $84 \%$ & 22 & $16 \%$ & 137 & \\
\hline
\end{tabular}

Fuente: Consejo Superior de Educación, 2005.

En el caso de las jefaturas de carrera, si bien la brecha de representación se estrecha, persisten diferencias notables entre varones y mujeres. El 67\% de estas jefaturas se encuentra en manos de varones, mientras que el 33\% restante en manos femeninas. La tabla 2 refleja la situación descrita. Al analizar datos relativos a la distribución jerárquica de docentes, el 20\% de los profesores eran titulares, de acuerdo con datos correspondientes a 1998, mientras que sólo el $6 \%$ de las profesoras lo eran. Mientras más baja la jerarquía mayor la proporción de mujeres; por ejemplo, entre profesores instructores el 20,8\% son mujeres y el 13,8\% son varones (Oyarzún, 2005).

Tabla 2: Jefes de carrera según género y título (INDICES pregrado 2005).

\begin{tabular}{l|r|r|r|r|c}
\hline & \multicolumn{2}{|c|}{ Hombres } & \multicolumn{2}{c|}{ Mujeres } & Total \\
\hline Técnicos & 910 & $65 \%$ & 495 & $35 \%$ & 1.405 \\
\hline Profesionales y/o licenciados & 1.831 & $68 \%$ & 856 & $32 \%$ & 2.687 \\
\hline Total & 2.741 & $67 \%$ & 1.351 & $33 \%$ & 4.092 \\
\hline
\end{tabular}

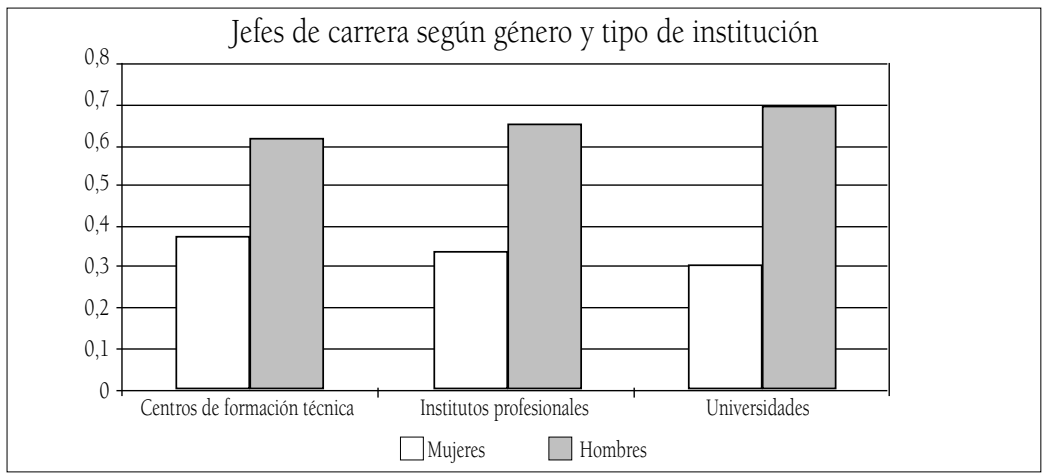

Fuente: Consejo Superior de Educación, 2005. 


\section{Aproximaciones al entramado de poder y género}

Las instituciones de educación superior son, por definición, heterogéneas en su composición y en sus metas sociales (Lolas, 1996), desembocando en una multiplicidad de significados de "poderes". Para los fines de este análisis, es conveniente comenzar comprendiendo el origen del poder. Una fuente de poder es la propiedad institucional, en donde la institución de educación superior pertenece a confesiones religiosas, grupos políticos o empresariales (Lolas, 2006). "En el caso de las universidades privadas, si bien no hay una representación masiva de mujeres rectoras, se dan unos pocos casos más. En estos casos, las mujeres han actuado como fundadoras de las universidades" (entrevistada 4). Alternativamente, la "tradición" se constituye como fuente de poder que se sustenta en quiénes están capacitados para ejercer funciones directivas. "Tradicionalmente, el rector sale por votación de adentro de la universidad. Se nombra un tribunal electoral, se presentan candidatos entre los docentes (...) cuando se utiliza el sistema tradicional de votación es más difícil que las mujeres lleguen al poder, porque hay hombres que han ascendido dentro de la estructura jerárquica de la universidad y están en posiciones más cercanas a la rectoría" (entrevistada 3). Asimismo, es posible señalar al consenso entre grupos de personas como fuente de poder (Lolas, 2006). “...Para llegar a este cargo tuve que pasar por un concurso, una elección y ahi tuve que hacer campaña, sacar una propuesta y presentarla a los académicos, a los funcionarios, a los estudiantes, estar en foros, generar consensos..." (entrevistada 1). Existe, adicionalmente, el poder derivado de fuerzas externas que actúan sobre las instituciones. "En general para ser rector de una universidad tienes que pertenecer a ciertos grupos que están dentro y fuera de la universidad" (entrevistada 3).

Como ya se comienza a detectar, las relaciones de poder son consustanciales a las de género. La presencia de la dimensión política ha permitido percibir la asimetría de dichas relaciones en un signo inequívoco de ejercicio de poder que implica el establecimiento de las relaciones de dominio. Si las relaciones de género implican a las de dominio, entonces hay que explicitar qué se entiende por poder o cuáles son los contenidos asociados. 
El poder existe sólo en el acto, constituyéndose como una relación que implica el ejercicio de fuerzas desiguales. "Entiendo el poder como la instancia en la cual uno puede influir en la toma de decisiones" (entrevistada 2); "Poder es hacer, servir, ser, influir. Para los hombres puede estar más asociado con jerarquía, dinero, importancia”. El poder produce cosas, induce placer, genera saber, produce discursos, es una red de relaciones productivas cuya función no es únicamente reprimir (Foucault, 1980; Ibáñez, 1982; Ceballos, 1987). Se aduce a la transversalidad del poder que hace a los sujetos sociales y a las mujeres, en lo particular, susceptibles de sufrir y ejercer poder como sujetos activos y no sólo pasivos de su historia personal. Desde la perspectiva de Foucault (1980), el poder transita transversalmente, no está quieto en los individuos. Esto potencia una visión de poder como un fenómeno fluido y dependiente de la situación social, que permite plantearse el sistema de género desde su capacidad de producción-reproducción y adaptación al contexto sociohistórico.

El género constituye un sistema ideológico que orienta las diferentes representaciones del sexo en función de exigencias culturales (Flores, 2001). "Las categorías hombre y mujer continúan siendo el locus de relaciones de poder que operan como espejo y reflejo de relaciones políticas, económicas y simbólicas que asignan un estatus y una valoración diferencial a lo femenino y a lo masculino, erigiendo así un andamiaje de desigualdades que se expresará en las diversas esferas en las que se construyen las subjetividades y las prácticas de los sujetos" (Montecino S., 2005: 1). En las complejas interacciones de género, las relaciones de poder son las más de las veces coercitivas. Las relaciones de poder en la práctica de las instituciones de educación superior parecen reproducir dichas relaciones. "...creo que el mundo académico es el mundo más machista que hay, lo que yo veo en el mundo académico tradicional particularmente es que es un mundo muy cerrado...” (entrevistada 2). Más aún, “... Los cargos altos los quieren los hombres y entre ellos se los quieren repartir, eso es verdad y uno por ser mujer claro que hay discriminación, creo que mucha discriminación es inconsciente... yo creo que los que están más 
arriba entre elegir una mujer y un hombre para ciertos cargos creo que les importa que se sea hombre, les da más confianza..." (entrevistada 3).

\section{Hacia un paradigma femenino del poder: marcando la carta de navegación de las mujeres en las instituciones de educación superior}

Estas instituciones han sido gobernadas por papas, príncipes, profesores, propietarios e, incluso, estudiantes (Brunner, 2005). En este contexto adquiere especial relevancia preguntarse sobre las cartas de navegación definidas por aquellas mujeres que han alcanzado posiciones directivas en tan prestigiosas instituciones.

El Programa de las Naciones Unidas para el Desarrollo (PNUD) (2004) asume que el poder actual de las mujeres es innegable, caracterizándolo como un mando inteligente, entretenido, no autoritario, amigable, de una conducción suave. Esta capacidad podría estar relacionada con el sentido femenino del sacrificio: las mujeres pueden renunciar a sí mismas para entregarse a los demás. En este mismo sentido, las posiciones directivas de educación superior se gestionan en manos femeninas con especificidades y particularidades. "... Las mujeres ejercemos el poder muy distinto de los hombres..." (entrevistada 2). Se tiene un sello de mucha participación, pero donde finalmente la decisión se toma en los equipos directivos. "Algo muy propio del estilo de gestión de las mujeres es que realizamos consultas constantes, pero la decisión la toma la rectora (...) el tipo de liderazgo que se tiene no es confrontacional, sino que catalizador y aglutinador” (entrevistada 3). “...Nosotras, las mujeres, somos más dialogantes, consultamos opiniones y lo hacemos de una manera femenina, consideramos las opiniones de los demás y nos colocamos más en el lugar del otro, utilizamos más esa posibilidad de empatizar con el otro..." (entrevistada 1).

El liderazgo es entendido como la capacidad, carisma o don para conducir y seducir voluntades en una organización; la capacidad para organizar equipos, contener y apoyar son relacionadas con 
características femeninas y las de mandar y resolver problemas con las masculinas. En este sentido, existe, talvez en forma clásica, un modelo autoritario de la gestión universitaria, muchas veces respaldada por la noción de que las tareas intelectuales son por definición jerárquicas y elitistas (Lolas, 2006: 41). Lo medular de este modelo es que el establecimiento de prioridades y control es dirigido desde arriba, con escasa retroalimentación por parte de los miembros de la comunidad educativa. Frente al modelo autoritario, se distingue un modelo participativo, cuyo tiempo decisional es más prolongado y exige mayor cantidad de argumentación (Lolas, 2006). En general, la mujer concibe al liderazgo como un medio de transformación de sus subordinados, aplica sus habilidades en las relaciones interpersonales para transformar el interés individualista por un objetivo colectivo, guiando como una totalidad a la empresa, nación u organización en la cual se desempeña.

Se alude incluso a un paradigma femenino. "Esto significa que surgen los afectos y emociones, pero esto no es un proceso racional, esto surge desde tu ontogénico, desde tu ser, esto sería parte de un paradigma femenino. El paradigma femenino partiría por reconocer que hay un paradigma masculino, con la imposición de que el hombre manda. El paradigma femenino implicaría trascender hacia lo emocional y afectivo, ser capaz de sonreír, andar como uno quiere, ser horizontal, etc. Este paradigma femenino apuntaría a que lo femenino también podría tener que ver con los hombres" (entrevistada 4). Este paradigma de poder incluiría como aspectos sustantivamente femeninos la diversidad, la solidaridad y el establecimiento de vínculos entre estas instituciones y la sociedad. "Hay una forma de acercarse a los problemas que hace que no existan miedos. Existe un ambiente de solidaridad y respeto a la diversidad, eso es un ambiente que rescata lo femenino" (entrevistada 4). "Las universidades necesitan vincularse mucho más con la sociedad y para eso las mujeres somos buenas, además las universidades tienen que aplanarse" (entrevistada 3). En este sentido, la perspectiva de género aporta una carta de navegación, un proyecto por armar que sugiere una universidad abierta a las ciudadanías y que se sustenta en epistemologías abiertas a las diferencias (Oyarzún, 2005). 
Se reconocen como imperativos estratégicos de la gestión de instituciones de educación superior algunas de las facetas definidas en los discursos de las mujeres que han alcanzado posiciones directivas. A saber, se sugiere fomentar la diversidad funcional y de conocimiento, la flexibilidad y un estilo de liderazgo participativo y colaborativo, en el cual se considere la opinión, parecer y sugerencias de la comunidad educativa en el proceso de toma de decisiones (Rodríguez, 2006).

Si bien las mujeres presentan una serie de habilidades y particularidades en su manera de gestionar el poder, el PNUD (2004) constata que no siempre se sienten cómodas en ese mundo. “...A veces me siento un poco rara, porque, por ejemplo, hoy día, que hubo consejo universitario y soy la única mujer entre 13 personas, más el rector y el prorrector que son hombres, entonces una se siente como pájaro medio raro (...) también me he sentido rara de que me hagan reverencias, a veces cuando yo llego a alguna reunión, como soy la única mujer todos se paran, lo tomo como una cosa simpática en todo caso, pero no ocurre lo mismo cuando llega un hombre..." (entrevistada 1). Por su parte, los hombres se muestran perplejos ante este nuevo dominio que experimentan en la vida cotidiana del trabajo y en la familia, “... hay una suerte de descolocación y lenta adaptación a un nuevo orden, el cual está todavía en plena constitución" (PNUD, 2004:112).

\section{El sistema de prestigio como elemento constitutivo del poder en las instituciones de educación superior}

Las entidades de educación terciaria se insertan en un lugar privilegiado del tejido social y cultural de los países, de modo que sus miembros gozan de un gran respeto y prestigio (Berríos, 2005). En este sentido, "las universidades son instituciones con un alto reconocimiento social, es muy prestigioso ser rector, por tanto los hombres están interesados en mantenerse en estas posiciones" (entrevistada 4).

Los requisitos básicos para acceder a posiciones directivas incluyen las habilidades de gestión y el "peso académico". "Para 
ser rectora tienes que "ser una buena carta", tienes que tener manejo administrativo y peso académico" (entrevistada 4). El sistema de prestigio adquiere relevancia como elemento constitutivo de poder en estas instituciones. Se alude al llamado "peso académico" que se definiría como "... tener una densidad, una trayectoria, ser reconocida por los pares (...) como alguien valioso que ha desarrollado una carrera académica, que ha ganado un prestigio por el trabajo académico que hace, ya sea en docencia, investigación, extensión...” (entrevistada 1).

En la lógica de los sistemas de prestigio, el llamado "peso académico" se refleja con especial énfasis en la posesión del grado de doctor, la participación en proyectos concursables, como los del Fondo Nacional de Desarrollo Científico y Tecnológico (FONDECYT) y FONDEF, y la publicación de revistas en Institute for Scientific Information (ISI) y en Scientific Electronic Library Online (SciELO), como algunas condicionantes para ascender en la estructura jerárquica a posiciones estratégicas. Los estándares del sistema de prestigio son parejos para hombres y mujeres, pero estas últimas presentan mayores dificultades para alcanzarlos. "...cuando se valoriza mucho el tema academicista, tenemos que reconocer que a las mujeres nos cuesta mucho más aceptar las becas de doctorado porque no podemos llegar y salir a estudiar fuera y dejar a nuestras familias o llevárnoslas." (entrevistada 3) "... si uno mira el número de profesores en esta facultad uno va a ver que alrededor de un 40\% del total de profesores somos mujeres, pero solo unas pocas llegan a la categoría de profesor titular y, por lo tanto, podrían llegar a sentarse a pensar en postular como candidata a decano, y entonces la pregunta es por qué sólo unas pocas llegan a ser profesoras titulares y ahi nos encontramos con que para llegar a ser profesor titular hay que trabajar muchísimo, hay que salir al extranjero, ir a congresos, estar permanentemente ganando proyectos, es un cuestión muy competitiva" (entrevistada 1). Estos planteamientos invitan a reflexionar acerca de las tensiones que experimentan las mujeres entre las responsabilidades familiares y laborales, entre lo privado y lo público. 


\section{Limitaciones y barreras en la carrera por el poder: tensiones entre lo privado y lo público}

La tensión entre trabajo y familia se entiende a partir de un progresivo distanciamiento entre la lógica del aprovechamiento de las ventajas competitivas que orienta el trabajo remunerado (mundo público) y la lógica de gratitud de la familia (mundo privado), que prescinde de cualquier cálculo y subordina la utilidad económica a la calidad de las relaciones humanas (Morandé, 2004). Uno de los mayores enfrentamientos entre lo público y lo privado se produce por el distinto valor que adquiere el tiempo en uno u otra, lo cual va acompañado por sentimientos de culpa o postergación del desarrollo profesional, en el caso de las mujeres que han accedido a cargos de poder. "...la verdad es que yo viví mis 10 primeros años de trabajo con un fervoroso cargo de conciencia, siempre de baja calidad, de estar en todos lados..." (entrevistada 2). "Vienen las culpas, pensamos en la casa, los hijos, la pareja y vemos que los roles caseros no se reparten. En este tipo de pegas tienes que ir a cócteles, actividades fuera del horario de trabajo" (entrevistada 3). "Las mujeres no nos atrevemos a abrir mundos asociados con el poder por el precio que tenemos que pagar, la familia. Nos postergamos por el bien familiar" (entrevistada 4).

Según Bourdieu (2000), el orden social funciona como una inmensa máquina simbólica que tiende a ratificar la dominación masculina en que se apoya. Es en este sentido que las mujeres viven en tiempos y espacios distintos a los hombres. Son ellas quienes laboran doblemente (en casa y fuera de ella); en general, se ubican en posiciones con bajos ingresos, carentes de poder y con escaso prestigio social (Reyes, 2006). Por un lado, las mujeres trabajan remuneradamente en mayor proporción que antes y, por otro, trabajan más horas que en décadas anteriores. En la práctica ello se traduce en la realización de una doble jornada de trabajo, dada la definición tradicional de los roles de género existentes en las familias chilenas (Márquez y Núñez, 1999). En la medida en que la creciente incursión de las mujeres en el trabajo remunerado no ha estado acompañada de una participación equivalente de los varones en la reproducción 
doméstica, se ha multiplicado la carga de trabajo que pesa sobre ellas (Ariza y de Oliveira, en Arriagada, 2004). “...Para la mujer ha sido más difícil poder llegar a estos cargos, como que ni siquiera nos planteamos la posibilidad de llegar a estos cargos porque requieren una dedicación bastante grande, casi extrema y (...) en nuestra sociedad a la mujer se la sigue viendo como la persona a cargo de la familia, a cargo de la casa y entonces eso hace que muchas se vayan quedando en el camino" (entrevistada 1). Por tanto, las mujeres que han accedido a posiciones de alta jerarquía en instituciones de educación superior experimentan, en alguna medida, la reproducción de la división sexual del trabajo, porque a la mujer se le asocia con el ámbito reproductivo-familiar y doméstico. Al hombre se le ve únicamente en la esfera productiva, casi como una especie de carga estructural-cultural, que refiere a un hombre para el cual trabajar es una obligación, por tanto, dejar de hacerlo o reducir su jornada no está dentro de sus posibilidades (Mauro y Godoy, 2001).

Por ahora, la incorporación de la mujer al mercado de trabajo no va acompañada por una distribución igualitaria del trabajo familiar, y los sistemas de representación simbólica masculinos apenas se han alterado. En este sentido, sólo las mujeres de clase media y alta podrán "ocultar" parte de la subordinación mediante la mercantilización del trabajo doméstico (Montecino, 2006). “...No habría podido hacer nada sin las personas que están en mi casa ayudándome. Son las nanas que trabajan en mi casa, ese es mi primer apoyo, porque yo vengo de un mundo bastante convencional, entonces mi marido no sabe hacer nada y no haría nada..." (entrevistada 2).

\section{Reflexiones finales}

La presencia de las mujeres en los más altos niveles de decisión y representación de las instituciones de la educación superior constituye un indicador de la equidad de género real y simbólico, contribuyendo al avance en otros ámbitos de la vida social. Como reflejo de una carta de navegación pospatriarcal a un nuevo trato social y laboral entre hombres y mujeres, la presencia de mujeres en 
las más altas posiciones de estas instituciones permitiría, al menos, abrir oportunidades simbólicas a otros hombres y mujeres, además de modelar comportamientos equitativos entre los(as) estudiantes de educación superior.

En el contexto posmoderno, en el cual la deshumanización se ha hecho cargo de los distintos aspectos de la vida humana, se hace imperativo romper el "cielo de vidrio" y permitir que más mujeres alcancen posiciones directivas, para poder ampliar el horizonte de soluciones y abrir un nuevo camino hacia la concreción de resultados diferentes, incluyendo las perspectivas y "voces" de las personas que forman parte de las instituciones de educación superior.

El sistema de prestigio adquiere relevancia como elemento constitutivo de la carrera académica y directiva en las instituciones de educación terciaria. Los estándares del sistema de prestigio son similares para hombres y mujeres, pero estas últimas presentan mayores dificultades para alcanzarlos. En este sentido, las tensiones entre las responsabilidades familiares y laborales, entre lo privado y lo público han sido manifestadas por las mujeres entrevistadas.

Cuando incorporamos la perspectiva de género al debate sobre educación superior y poder, se abren una serie de nuevas interrogantes para futuras investigaciones y estudios. Las entrevistadas enuncian algunas de estas preguntas: “PPor qué hay pocas mujeres en el poder? ¿Quiénes son los poderosos? ¿Habrá poderosos que impidan que las mujeres lleguen a posiciones estratégicas en las instituciones de educación superior, o serán las mujeres las que no quieren llegar a posiciones de poder en las universidades? (entrevistada 4). "¿Cuántas mujeres hay en el sistema universitario y en qué posiciones se encuentran? ¿Por qué están en dichas posiciones? ¿Quieren seguir ascendiendo? ¿Cuáles serían las barreras de pasar de una posición cercana a la rectoría a ser rectora?" (entrevistada 3). "¿Cuáles son las barreras que obstaculizan que la mujer siga avanzando y que pueda llegar a cargos de poder similares a los del hombre? ¿Qué se puede hacer para ayudar a superar esas barreras?" (entrevistada 1). 
Este documento ha cumplido con abrir espacios a nuevas interrogantes y reflexiones en torno al tema. La invitación es a generar líneas de investigación, proyectos de intervención y planificación de políticas públicas e institucionales que permitan avances en las relaciones de poder y género.

Agradezco muy especialmente la colaboración de mi ayudante de investigación, María Alicia Sebastián, Trabajadora Social y estudiante del Magíster en Trabajo Social de la Pontificia Universidad Católica de Chile.

\section{Referencias bibliográficas}

Arriagada, Irma y Aranda, Verónica (comp.) (2004) Cambio de las familias en el marco de las transformaciones globales: necesidad de políticas públicas eficaces. Santiago de Chile: División Desarrollo Social. ONU-CEPALUNFPA.

Becher, T. (2001) Tribus y territorios académicos. La indagación intelectual y la cultura de las disciplinas. Barcelona: Gedisa.

Berríos, Paulina (2005) El sistema de prestigio en las Universidades y el rol que ocupan las mujeres en el mundo académico. Calidad en la Educación (23): 449-361.

Bourdieu, Pierre (2000) La dominación masculina. Barcelona: Anagrama, Colección Argumentos.

Brunner, José Joaquín (2005) Gobierno Universitario. El Mercurio, 22 de marzo.

Ceballos, H. (1997) Foucault y el Poder. México: Ediciones Coyacán.

Correa, M. y Monckeberg, M. (2001) Mujeres Chilenas. Estadísticas para el Nuevo Siglo. Santiago de Chile: SERNAM e INE.

Doña, Karina (2004) Liderazgo femenino, ¿mito o realidad? 4 (5). Agenda pública.

Fisher, H. (2000) El Primer Sexo: las capacidades innatas de las mujeres y cómo están cambiando el mundo. Madrid: Editorial Taurus.

Foucault, M. (1980) Microfísica del Poder. Madrid: La Piqueta. 
Flores, F. (2001) Psicología social y género. El sexo como sujeto de representación social. México: McGraw Hill.

Fundación Futuro (2003) Mujeres en Chile/Percepción. [Sitio en Internet] Disponible en http://www.comunidadmujer.cl/cm/mujeresencifra/ Omujcifras.asp

García, Adela (ed.) (2004) Género y ciudadanía. Un debate. Barcelona: Icaria Editorial.

Guil, Ana (2002) El Viaje de Penélope: Vicisitudes de las Mujeres en el Mundo Académico. El Telar de Ulises, 2.

Ibáñez, T. (1982) Poder y Libertad. Barcelona: Editorial Hora.

Lerena, C. (1976) Escuela, ideología y clases sociales en España. Barcelona: Ariel.

Lolas, Fernando (1996) Las universidades chilenas y sus entornos: dilemas y problemas. En Riveros, L. y Sapag, N. (eds.) Retos y Dilemas de la Gestión Universitaria. Santiago de Chile: Universidad de Chile, Facultad de Ciencias Económicas y Administrativas, pp: 45-56.

Lolas, Fernando (2006) Sobre modelos de gestión universitaria. Calidad en la Educación, 24: 37-45.

Márquez, Francisca y Núñez, Lorena (1999) Compatibilidad entre vida laboral y vida familiar: experiencias y políticas. Sitio Sur, 22.

Mauro, Amalia y Godoy, Lorena (1999) Cambios en el mercado de trabajo y relaciones de pareja: el punto de vista de los hombres. Santiago de Chile: Centro de Estudios de la Mujer-CEM. [Sitio en Internet] Disponible en http://www.cem.cl/pdf/cambio_mercado.pdt

Maruani, M y otros (2000) Las nuevas fronteras de la desigualdad. Barcelona: Icara.

Monserrat, Silvia Inés y Saracostti, Mahia (2006) The Successful Women of Chile. In: Punnett, Betty Jane et al. Successful professional women of the Americas: from polar winds to tropical breezes. Cheltenham, UK; Northampton, MA: Edward Elgar.

Montecino, Sonia (2005) Identidad de Género, entramado e igualdad de poder. Ventana Académica. Universidad de Chile.

Morandé, Pedro (2004) Sociedad, trabajo y familia: un mundo, dos lógicas. En: Errázuriz y Riedemann (eds.) Construir Sentido: Sociedad, Familia y Trabajo. Santiago de Chile: Servimpress. 
Papadópulos, Jorge y Radakovich, Rosario (2006) Informe sobre la educación superior en América Latina y el Caribe. Capítulo 8: Educación Superior y Género en América Latina y el Caribe. UNESCO-IESALC, pp: 117128.

Parella, S. (2003) Repensando la participación de las mujeres en el desarrollo desde una perspectiva de género. Barcelona: Universidad Autónoma de Barcelona, Departamento de Sociología, Paper 69.

PNUD (2004) El poder: ¿Para qué y para quién? Santiago de Chile: Desarrollo Humano en Chile.

Reyes, Jenny (2006) Trabajadores (as) de la educación superior y reproducciones de género. Calidad en la Educación, 24, pp. 399-442.

Rodríguez, Emilio (2006) El proceso de toma de decisiones estratégicas en las universidades públicas. Calidad en la Educación, 24., pp. 49-63.

Oyarzún, Kemy (2005) Un trato de las diferencias: Género y Educación Superior en Chile. Universidad de Chile. Documentos CSE, pp. 1-24.

Salazar, José Miguel (2005) Educación superior y género. Tendencias observadas. Documentos CSE, Chile.

Selame, Teresita (2004) Mujeres, Brechas de Equidad y Mercado de Trabajo en Chile. Santiago de Chile: OIT y PNUD.

Taylor, Steven y Bogdan, Robert (1992) Introducción a los métodos cualitativos de investigación. Madrid: Paidós.

Wirth, L. (2002) Romper el techo de cristal: las mujeres en puestos de dirección. Madrid: Colección Informes OIT N58.

Recibido: 19 de octubre de 2006

Aceptado: 2 de noviembre de 2006 